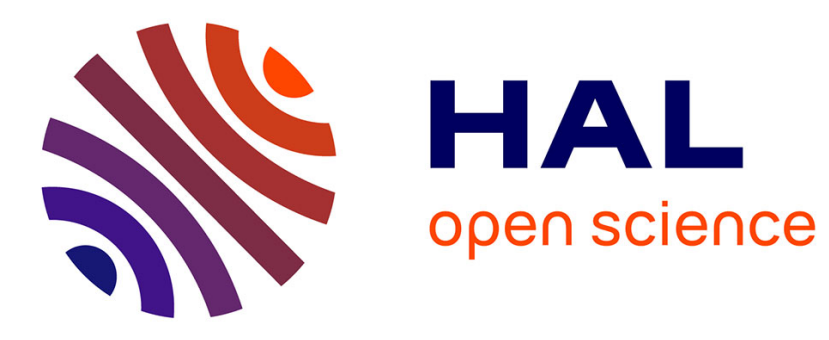

\title{
Energy Efficient Channel State Classification for Lifetime Enhancement of LPWA Networks
}

\author{
Chhayarith Heng Uy, Carolynn Bernier, Sylvie Charbonnier
}

\section{To cite this version:}

Chhayarith Heng Uy, Carolynn Bernier, Sylvie Charbonnier. Energy Efficient Channel State Classification for Lifetime Enhancement of LPWA Networks. COMSNETS 2019 - 11th International Conference on COMmunication Systems \& NETworkS, Jan 2019, Bangalore, India. 10.1109/COMSNETS.2019.8711438 . cea-01972976

\section{HAL Id: cea-01972976 https://hal-cea.archives-ouvertes.fr/cea-01972976}

Submitted on 8 Jan 2019

HAL is a multi-disciplinary open access archive for the deposit and dissemination of scientific research documents, whether they are published or not. The documents may come from teaching and research institutions in France or abroad, or from public or private research centers.
L'archive ouverte pluridisciplinaire HAL, est destinée au dépôt et à la diffusion de documents scientifiques de niveau recherche, publiés ou non, émanant des établissements d'enseignement et de recherche français ou étrangers, des laboratoires publics ou privés. 


\section{Energy Efficient Channel State Classification for Lifetime Enhancement of LPWA Networks}

\author{
Chhayarith Heng Uy \\ Univ. Grenoble Alpes \\ Gipsa-Lab, CEA, LETI \\ Grenoble, France \\ chhayarith.heng-uy@univ-grenoble-alpes.fr
}

\author{
Carolynn Bernier \\ Univ. Grenoble Alpes \\ CEA, LETI \\ Grenoble, France \\ carolynn.bernier@cea.fr
}

\author{
Sylvie Charbonnier \\ Univ. Grenoble Alpes \\ Gipsa-Lab, CNRS \\ Grenoble, France \\ sylvie.charbonnier@univ-grenoble-alpes.fr
}

\begin{abstract}
Current Low Power Wide Area Network (LPWAN) wireless transceivers are designed, or configured at deployment time, to function assuming a worse-case application scenario. Most of the time, they waste a significant amount of energy when operated under favourable channel conditions. Energy efficient and accurate channel state classification is imperative for selecting the optimum trade-off between transceiver performance and amount of saved energy, without impacting transmission quality. This work presents a novel, low complexity channel state indicator and a simple mono-feature classifier for channel state recognition. The classifier is trained using a set of experimental data acquisitions and has a $96 \%$ accuracy when tested with a new collected data set. Specially designed for LPWA applications, the classifier is capable of distinguishing undisturbed channels from those suffering from both mobility-induced fading and interference, while operating at low to medium SNR.
\end{abstract}

\section{INTRODUCTION}

The ultimate goal in the field of the Internet of Things (IoT), and, more specifically, of Low Power Wide Area (LPWA) wireless networks, is the development of strategies to reduce the average energy consumption of the autonomous network nodes. To this end, the automatic adaptation of the transceiver performance to the varying Radio Frequency (RF) channel is a research perspective whose aim is to eliminate the unnecessary energy consumption in moments when the signal to noise ratio (SNR) is in excess with respect to the communication link budget. Indeed, a lot of recent work has focused on the many tuning knobs that can be used to trade excessive SNR against energy consumption, e.g. output power, modulation order, coding rate, frame length, receiver noise figure, ... However, in all cases, to enable any channel state adaptation algorithm, it is necessary to develop energy efficient channel state recognition mechanisms.

Wireless sensor nodes using LPWA communication technologies represent a large fraction of IoT networks. These nodes are typically deployed for low mobility, km-range applications. The large distances involved imply that the RF communication channel will have a wide dynamic range, making it necessary, and particularly for systems employing ISM bands, to identify at least three channel states:

- An Undisturbed state where noise can be modelled as a white Gaussian random process;
- An Interference state in which simultaneous RF transmissions can impede the desired transmissions;

- A Fading state in which the transmission quality is impacted by the movement of the node itself or of humans or objects in the node's environment.

The identification of these three channel states, versus 2-state approaches which are commonly proposed in the literature, makes it indeed much easier to develop adaptation strategies that are specific to the observed propagation conditions.

Due to the large distances, the main energy cost for LPWA nodes is the one required for wireless transmissions. Any channel state identification mechanism must therefore avoid the transmission of dedicated frames or symbols or, in case of erroneous channel classification, of repeated frames. In addition, the calculation complexity of the channel state indicator (CSI) must be low to avoid the power burden on the wireless node's application processor. If we wish to ease the development of new MAC or network-level adaptation algorithms, the CSI must be clear and simple to use, with ideally a single value computed per frame. And finally, since the future channel-aware system is meant to function at low to medium SNR, the CSI must perform at its best in these situations.

This paper focuses on the development of low complexity channel analysis signals (CAS) which provide relevant features for accurate channel state classification. While this present work is limited to the LECIM (Low Energy Critical Infrastructure Monitoring) FSK PHY of the IEEE 802.15.42015 standard, our aim is to show that such an approach can be generalized to other LPWA standards such as LoRa, and including cellular standards such as NB-IoT. With this demonstration, our hope is to encourage the development of future transceivers that natively embed such a capability in order to enable the massive deployment of channel-aware protocols.

The contributions of this paper are:

- To the authors' knowledge, the first channel state classifier tailored to the requirements of LPWA networks with a specific focus on the life-time enhancement of energy autonomous nodes; 
- A 3-state channel identification algorithm adapted to low to medium SNRs built upon a low complexity and single frame classifier with $96 \%$ accuracy.

- An experimentation-based classifier design methodology which can be easily generalized to other LPWA communication standards.

This paper is organized as follows: the specifications of the desired classifier are given in Section II, followed by related work in Section III. Our design methodology and proposed approach are presented in Section IV and V, respectively. Experimental results are discussed in Section VI.

\section{Classifier Requirements}

Our aim is to design a channel state indicator dedicated to LPWA networks in which application throughput is typically very small and where the most important requirement is improving node lifetime and not the throughput of the wireless link. For heavily duty-cycled IoT applications in which frame transmissions are relatively 'rare', single-frame channel diagnostics is a must. The orders of magnitude energy cost difference between RF data transmissions and local, on-board data processing implies that the long periods between successive frame transmissions can be leveraged for computations that can be performed at low clock rates. Our goal is therefore to design a simple to use, low complexity, information-rich channel state indicator (CSI) able to ease the development of high performance adaptation protocols. Indeed, the more precisely we can describe the instantaneous channel conditions, the better the adaptive protocol (whether at MAC or routing-level) can make appropriate reconfiguration decisions. The proposed classifier will have the capacity to discriminate between three channel classes: 'undisturbed', 'fading', when the channel is impacted by mobility-induced fading, and 'interference', when simultaneous RF transmissions are detected.

Additionally, the classifier must be accurate in low to medium SNR situations since they are those in which the future channel-aware system is meant to function (in steadystate). Recognizing the channel state at low to medium SNR is imperative to save a maximum of energy without impacting transmission quality. In particular, it is imperative to know the channel state before the onset of symbol errors (due to channel imperfections) if the frame is un-coded. Finally, the classifier must not become 'blind', i.e. incapable of recognizing interference or fading states, at medium SNR levels.

\section{RELATED WORK}

Practically all previous work on channel quality recognition aimed at either increasing the network throughput or improving the reliability of routing protocols. A huge body of work exists in which authors employ indicators available on Commercial Off The Shelf (COTS) radio transceivers, such as received signal strength indicator (RSSI) or link quality indicator (LQI), or MAC-level metrics such as packet reception ratio (PRR), and so on [1]. However, many authors have discussed the limited accuracy and potentially misleading nature of such metrics [1]-[3]. More generally, using these metrics, it is often difficult to understand the reasons behind the loss of channel quality. And metrics requiring many transmitted frames (such as PRR) necessarily miss short-term variations of channel quality.

The inefficiencies of these methods have motivated recent work on finer-grain approaches able to discriminate between different types of channel impairments [2], [4]-[6]. In [2], software defined radios programmed as IEEE 802.15.4 [7] receivers with hard chip decisions were instrumented to calculate the per-symbol Hamming distance between the received chip sequences and the predefined pseudo-random codes. A pattern matching analysis of this signal is performed for several consecutive frames and the cumulative result feeds a classification algorithm that distinguishes between normal (undisturbed), attenuation (due to the propagation medium, reflection and propagation distance) and interference types of channels.

The work in [2] is a special case of a more general approach that was presented earlier in [4] and [5]. Here, the higher protocol layers are provided not only with the frame's

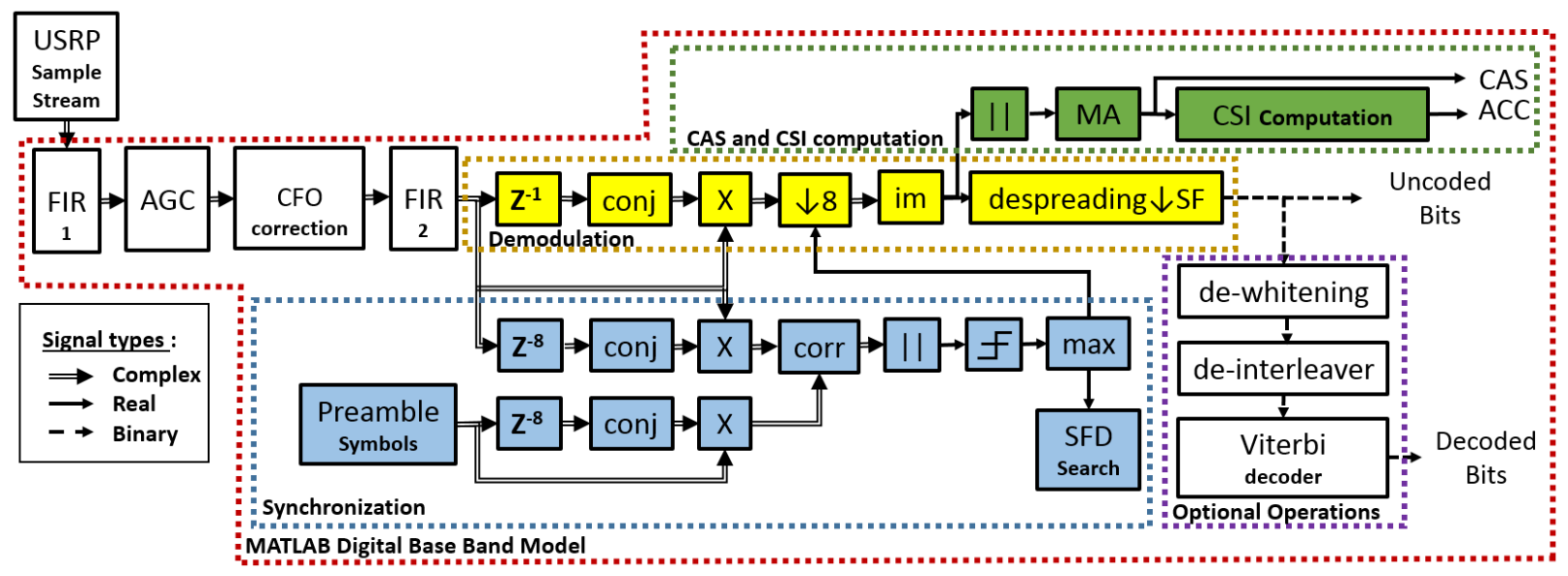

Fig. 1. IEEE 802.15.4-2015 LECIM FSK PHY digital baseband receiver algorithm with CAS and CSI computation. 
decoded symbols, but also with information corresponding to the probability that each decoded symbol is correct, termed SoftPHY hints by the authors. Using this information, a persymbol SNR can be estimated precisely. Sudden jumps in SNR can be identified as frame collisions. As discussed above, the SoftPHY hint for a symbol can be simply the Hamming distance separating the decoded and ideal symbols in a hard decision decoding spread spectrum receiver or it could also be the modulus of the log likelihood ratio that is produced by certain linear convolutional or block decoders. However, since all of these SoftPHY hints (one per symbol) are passed to the higher layers without further processing, the authors assume that it is up to the higher layers to do the job of signal analysis and classification.

In [6], the authors use forward error correction (FEC) on top of (normally un-coded) IEEE 802.15.4 transmissions in order to extract the position of bit errors within each frame. Using this information, the authors compute a symbol error density (SED) defined as $c /\left(S_{L}-S_{F}+1\right)$, where $c$ is the number of detected symbol errors, and $S_{L}$ and $S_{F}$ are respectively the last and first error positions. Experiments in the presence and absence of interference are used to find a discriminating threshold for a binary classifier. While this technique has the important advantage of being applicable to COTS transceivers, the coding overhead results in a significant power consumption penalty. In addition, only two channel states are defined, limiting the possibilities offered to the adaptation protocol. We include a detailed comparison of the SED-based classifier with our work in the results below.

\section{Experimentation-BAsed Methodology}

An experimentation-based methodology is used to design our classifier in order to ensure that it can correctly handle real-world signals, as opposed to signals which would be constructed only through simulation. While this methodology is applicable to any LPWA wireless communication system, for this work, we choose to implement radio transceivers based on a variant of the IEEE 802.15.4-2015 LECIM FSK PHY standard [7] which is an LPWA standard dedicated to critical infrastructure monitoring. After a description of this standard, the SDR experimentation platform is described.

\section{A. DBB Receiver for IEEE 802.15.4-2015 LECIM FSK PHY}

This variant of the LECIM FSK PHY employs the GMSK modulation at $37.5 \mathrm{kchip} / \mathrm{s}$. The frame structure is illustrated in Fig. 2. The preamble is a "00110011" four byte sequence. The 16-bit CRC sequence is based on the generator polynomial $G(x)=x^{16}+x^{15}+x^{2}+1$. FEC can optionally be used and is a rate $1 / 2$ convolutional coding with constraint length $K=7$. The LECIM FSK PHY optionally uses direct-sequence spread spectrum modulation with a spreading factor from 2 to 16. If FEC encoding is used, only the payload and the CRC are coded, interleaved, whitened and optionnally spread. The header is treated similarly but without the whitening step. The preamble and the SFD are added as is to the beginning of the frame. In the channel state classifier design described below, the use of FEC is not necessary but we have implemented the convolutional coding/decoding step to allow the computation of a SED for performance comparisons.

\begin{tabular}{|c|c|c|c|c|}
\hline \multicolumn{5}{|c|}{ Octets } \\
\hline 4 & 3 & 2 & 1250 & 2 \\
\hline Preamble & SFD & Header & Payload & CRC \\
\hline
\end{tabular}

Fig. 2. Implemented frame structure (compatible with IEEE 802.15.4-2015 LECIM FSK PHY).

The corresponding DBB receiver is presented in Fig. 1. Blocks in blue handle frame synchronization and start of frame delimiter (SFD) detection. The synchronization algorithm, based on a correlation with the known preamble sequence, also finds the optimal decimation moment, allowing the sample stream to be decimated by a factor of 8 . Blocks in yellow perform demodulation while blocks in green compute the channel analysis signal (CAS) which is used to extract the channel state indicator (CSI). The DBB is composed of the following elements:

- FIR1 is a wide band, high order low pass filter with a -3 $\mathrm{dB}$ cut-off frequency $f_{F I R 1}=65.7 \mathrm{kHz}$. The value of this cut-off frequency takes into account the chip-rate, the maximum carrier frequency offset (CFO) of the hardware and a safety margin;

- AGC is an automatic gain control block which outputs a controlled amplitude signal. This block simplifies the synchronization algorithm of the DBB by allowing the use of a constant preamble detection threshold;

- The CFO block calculates and corrects the carrier frequency offset;

- FIR2 is a low pass, high order filter with a -3 dB cut-off frequency $f_{\text {fir } 2}=30.7 \mathrm{kHz}$.

- Next, the Hermitian product between samples $A[k]$ and $A[k-1]$ is calculated. The Hermitian product is defined as $A[k] \times A^{*}[k-n]$ with $n$ the delay between the two samples. With a delay of 1 , the imaginary part of the product is a good approximation of the instantaneous frequency and can therefore be used to demodulate FSK signals.

- Finally, the imaginary part of the signal is the soft input to the de-spreading block.

Fig. 3 shows the simulated BER performance of the implemented IEEE 802.15.4-2015 LECIM FSK PHY baseband receiver. The ExpSF4 series are measurements made using the SDR-based platform described below.

\section{B. SDR-Based Experimentation Platform}

Since COTS devices do not allow access to DBB algorithms, an SDR platform is needed for experimentation. The goal of the experiments is to provide realistic data for the classifier training set and the test set. Physically, the SDR platform is composed of three universal software radio peripherals (USRP) placed in a $5 \mathrm{~m} \times 4 \mathrm{~m}$ room. The first one is used as transmitter $(T x)$, the second as receiver 


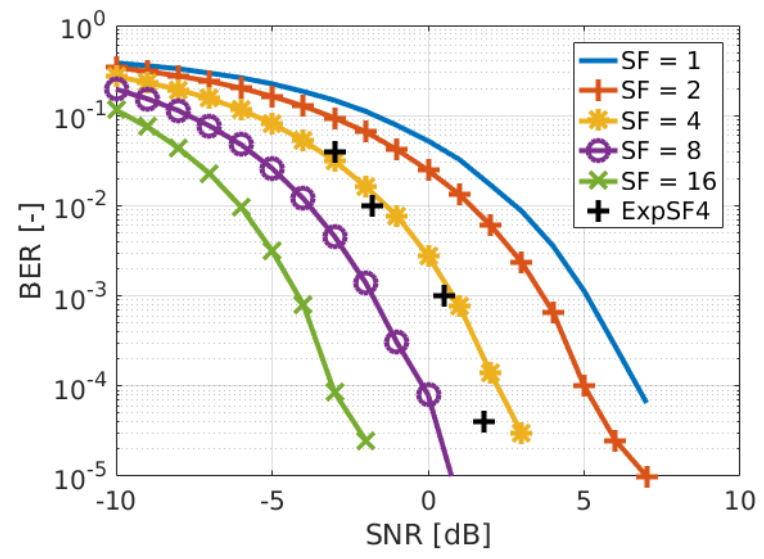

Fig. 3. Bit Error Rate performance of the implemented IEEE 802.15.4-2015 LECIM FSK PHY baseband receiver. The ExpSF4 series is obtained from the SDR-based experimentation platform.

$(R x)$ and the third as the interferer $(I x)$. The USRPs are N210 models [8] from Ettus Research with WBX [40 MHz - $2200 \mathrm{MHz}$ ] daughter-board [9]. All experiments employ a carrier within the 869.3-869.4 MHz ISM band which is not limited by duty cycle in France, if transmissions are below $10 \mathrm{~mW}$. Since we are interested in studying the behaviour of the receiver in low SNR conditions, attenuators are inserted between each emitting USRP output SMA port (60 dB for $T x$ and $30 \mathrm{~dB}$ for $I x$ ) and the half-wave dipole antenna. The interferer $I x$ frequency carrier has an offset of $100 \mathrm{kHz}$ from that of $T x$. This allows us to limit the transmission range to approximately two meters which is necessary for leading controlled experiments.

Each USRP is connected to a PC by Gbit Ethernet. Each USRP is driven by GnuRadio and acts as an RF front-end with up/down frequency conversion, analog filtering and DA/AD conversion. For the $T x$ and $I x$, complex baseband data generated by MATLAB are fed to the USRP by GnuRadio. For the experiments, 1250 byte length frames were used in order to capture long duration signals which are typical of LPWA frames at maximum coupling loss (2 seconds here). Frames were generated with a spreading factor of 4 and encoded as described previously. For the $R x$, the USRP samples the baseband complex sample stream at $300 \mathrm{kHz}$, i.e. 8 samples per symbol, which is then fed to the MATLAB DBB.

\section{Data collection}

In order to produce labelled data-sets, three experiments are executed. In the first, frames are acquired in an undisturbed environment. For the second, an interference environment is generated using $I_{x}$. To avoid wrongly labelled frames, we ensure that all received frames are disturbed by running $I_{x}$ with a duty cycle of $0.4 \mathrm{~s}$. We limit our experiments to interferers of inferior frame length with respect to the desired frame since the opposite case can be identified as a sudden change in SNR. The interferer employed in our experiments is an identical IEEE 802.15.4-2015 LECIM FSK PHY transmitter. Only constant envelope interferers are considered (e.g., FSK-based transmissions, LoRa) since these are the most common in LPWA communications. The third experiment is executed in a mobility context, including pedestrian motion near the USRPs, or an arm or object moving trough the direct transmission path between $T x$ and $R x$. All signal acquisitions are made at low to medium SNR. The variation of SNR is obtained by changing the Tx USRP RF gain. A total of 360 frames are collected,120 for each experiment and labelled 'undisturbed', 'interference' or 'fading'. This is enough for feeding the classifier training and evaluation steps. Only frames with valid CRC were analyzed.

\section{Low Complexity Channel State Classification}

In this section, we present the low complexity channel analysis signal (CAS) and channel state indicator (CSI) that were selected to build our channel state classifier.

\section{A. Channel Analysis Signal}

Similarly to previous work by [2], [4], [5], we directly exploit signals present in the digital baseband receiver to extract a low complexity CAS. The CAS must reflect the certainty level that the received symbol is correct. In GMSK, the symbols (corresponding to chips in the IEEE802.15.4-2015 LECIM FSK PHY) are represented by frequency deviations of the signal. The standard deviation of the absolute value of the instantaneous frequency estimations, already calculated in the DBB thanks to the Hermitian product, is proportional to the uncertainty level of the received chips (and therefore, inversely proportional to SNR). The absolute value of the imaginary part of the Hermitian product has the additional advantage of being robust to fluctuations of receive signal amplitude.

With $x_{k}, k=1, \ldots N$ the outputs of the imaginary part extraction block (cf. Fig.1), a $C A S_{k}$ is computed for each received chip, where

$$
C A S_{k}=\frac{1}{w i n} \sum_{i=0}^{\text {win-1 }}\left|x_{k-i}\right| .
$$

We define $C A S=\left[C A S_{1}, \ldots, C A S_{N}\right]$ as the channel analysis signal. To compute each $C A S_{k}$, a moving average over a computation window of win samples is used to smooth the signal and highlight the disturbance footprint. The value win $=1000$ is selected which corresponds to a duration of $T_{w i n}=26.6 \mathrm{~ms}$ given the $37.5 \mathrm{kHz}$ sample rate. The choice of win is compatible with the duration of the expected RF interference as well as the expected mobility of objects or humans in the vicinity of the nodes.

Figures 4, 5 and 6 show examples of the CAS computed on frames transmitted through channels labelled as 'undisturbed', 'interference' and 'fading'. Observing the curves we can see the temporal footprint corresponding to each channel state. For the 'undisturbed' case, the CAS has a small amplitude and fast variations around the average value, $\mu_{C A S}$. In those labelled as 'interference', the presence of the interferer is clearly visible. In those labelled as 'fading', the duration of the 
fading phenomena can clearly be put into perspective with the coherence time $T_{c}$ of the channel, i.e. the time during which two transmissions suffer correlated fading effects, defined as $0.4 * c /(v f)$, where $v$ is the speed of the moving element, $f$ is the carrier frequency, and $c$ is the speed of light [10]. Assuming a sub-GHz carrier, a coherence time on the order of $100 \mathrm{~ms}$ is typical of low mobility IoT use-cases. The complexity of the CAS computation is very low, requiring only a modulus computation and a moving average filter in the DBB.

The CAS computation is easily generalized to other standards. E.g. in a LoRa DBB, symbols are recovered as bin locations of an FFT performed on the received sample stream. [11]. In this case, the CAS could be defined as the magnitude of the FFT bin corresponding to the LoRa symbol.
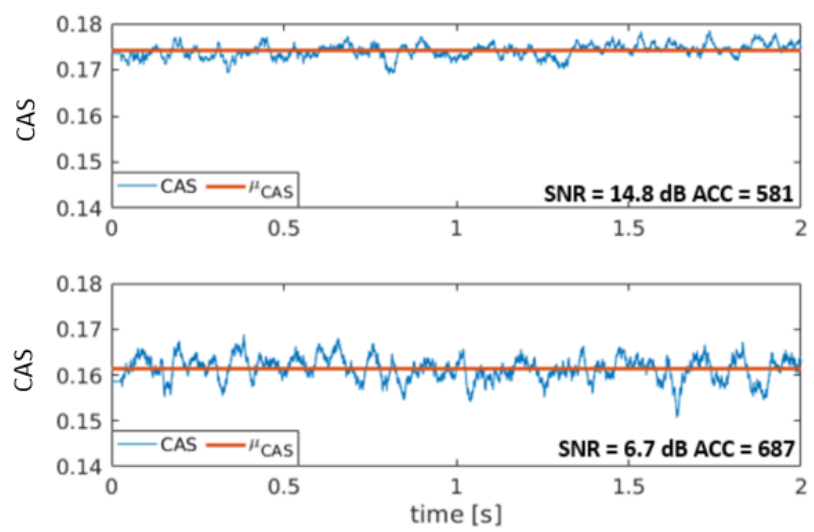

Fig. 4. CAS and ACC for two frames transmitted through 'undisturbed' channel
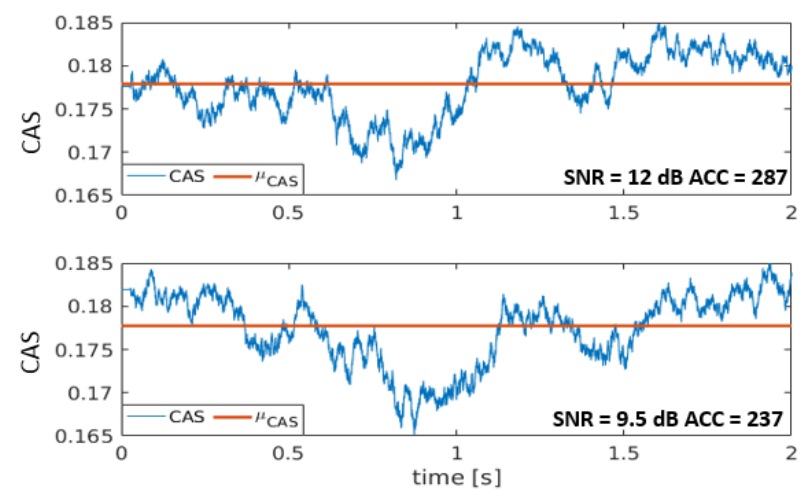

Fig. 5. CAS and ACC for two frames transmitted through 'fading' channel

\section{B. Low Complexity Channel State Indicator}

We propose a very simple and low complexity CSI computed from the CAS defined above which condenses the state channel information into only one value. We define our CSI as the average crossing count (ACC) which captures the variations of the CAS around its average value. An ACC value is computed for each received frame which can be fed to the classification algorithm to identify the current channel state.

To compute ACC, the CAS is centered by its average $\mu_{C A S}$ to convert the average crossings to zero crossings. The average
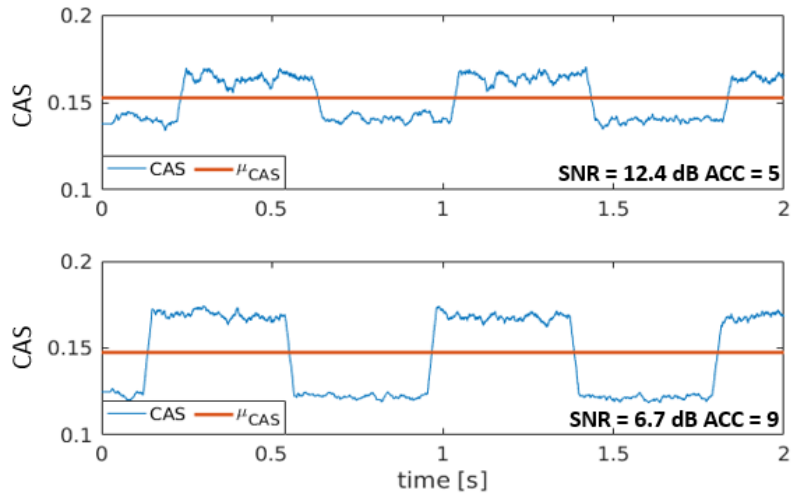

Fig. 6. CAS and ACC for two frames transmitted through an 'interference' channel

crossing count is the summation of the $S_{k}$ sign switches, described by (3). The CSI computational complexity is low and the size of required memory to temporarily save the CAS values depends on the maximum frame length. Fig. 8 shows the labelled ACC distribution according to the different channel states.

$$
\begin{gathered}
S_{k}=\operatorname{sgn}\left(C A S_{k}-\mu_{C A S}\right) \\
A C C=\frac{1}{2} \sum_{k=2}^{N}\left|S_{k}-S_{k-1}\right|
\end{gathered}
$$

\section{Classifier Definition and Training}

In this work, the classification in the three channel classes, 'undisturbed', 'fading' and 'interference', is done using a single feature, ACC. Two thresholds, $\lambda_{1}$ and $\lambda_{2}$, are required to discriminate between the three classes. The channel state is classified as 'interference' when the ACC is below $\lambda_{1}$, as 'undisturbed' when ACC is higher than $\lambda_{2}$ and as 'fading' otherwise. The value of $\lambda_{1}$ and $\lambda_{2}$ are set using a learning data set consisting of 180 randomly chosen labelled frames acquired using the SDR platform. Each class, 'undisturbed', 'fading' and 'interference', contains 60 frames. To evaluate the ability of ACC to discriminate between the 'undisturbed' and 'disturbed' (fading and interference) channel states and then between the 'fading' and 'interference' states, ROC curves are built. Let us consider the 'undisturbed'/'disturbed' detector case. A frame with an ACC higher than $\lambda_{2}$ is classified as 'undisturbed', otherwise it is classified as 'disturbed'. The ROC curve displays the true positive rate (the percentage of 'disturbed' frames correctly classified as 'disturbed') as a function of the false positive rate (the percentage of 'undisturbed' frames classified as 'disturbed') for various values of $\lambda_{2}$, varying from 0 to the maximum value of ACC.

Fig. 7 shows the ROC curves for the 'undisturbed'/'disturbed' and 'fading'/'interference' detectors using the learning data set. One can see that ACC proves to be quite efficient in discriminating between 'disturbed' and 'undisturbed' frames as well as between 'interference' and 'fading' frames. The two ROC curves almost reach the 
performance of the ideal detector i.e. $100 \%$ TP with $0 \%$ of FP. The two ROC curves can be used to select the value of the two detection thresholds $\lambda_{1}$ and $\lambda_{2}$. Both are selected from the optimum operating point i.e. the point closest to $(0,1)$, the ideal performance. The optimum operating points provide the $\lambda_{1}$ and $\lambda_{2}$ thresholds of 85 and 530, respectively. Fig. 8 shows the labelled training set ACC distribution with respect to $\lambda_{1}$ and $\lambda_{2}$. We can observe that these thresholds provide good boundaries between the three classes. Since the ROC curves never pass by the $(0,1)$ point, the classifier is not flawless thus explaining the presence of some error points in the ACC distribution.
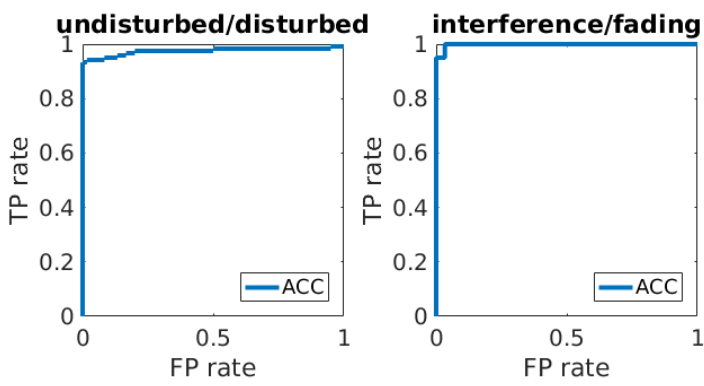

Fig. 7. ROC curves for classifying, respectively, undisturbed versus disturbed channel states (left) and interference versus fading channel states (right).

Finally, the classifier tuned on the learning data set is expressed as follows :

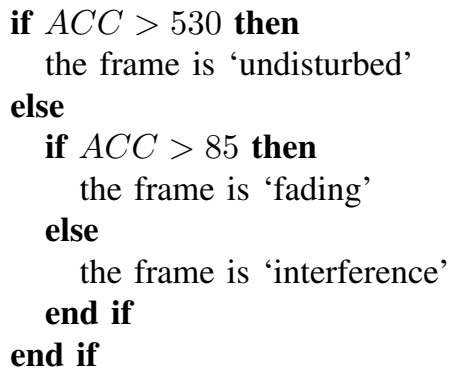

\section{EXPERIMENTAL RESULTS}

The performance of the classifier is evaluated on a validation set, made of the 180 labelled frames acquisitions (60 frames per class), which were not used in the training set. The ACC-based classifier is able to correctly classify $96 \%$ of the 180 frames, 3 'undisturbed' frames were misclassified as 'fading' and 5 'fading' as 'undisturbed'.

Next, we compare our ACC-based CSI with the symbol error density (SED) indicator presented in [6]. Recall that this indicator requires forward error correction (FEC) in order to extract the position of bit errors within each frame. The SED is defined as $c /\left(S_{L}-S_{F}+1\right)$, where $c$ is the number of detected symbol errors and $S_{L}$ and $S_{F}$ are respectively the last and first error positions. The authors claim that comparing the SED to a simple threshold is sufficient to distinguish between two channel classes: an interference class and a class where channels suffer from multi-path fading or attenuation (MFA).

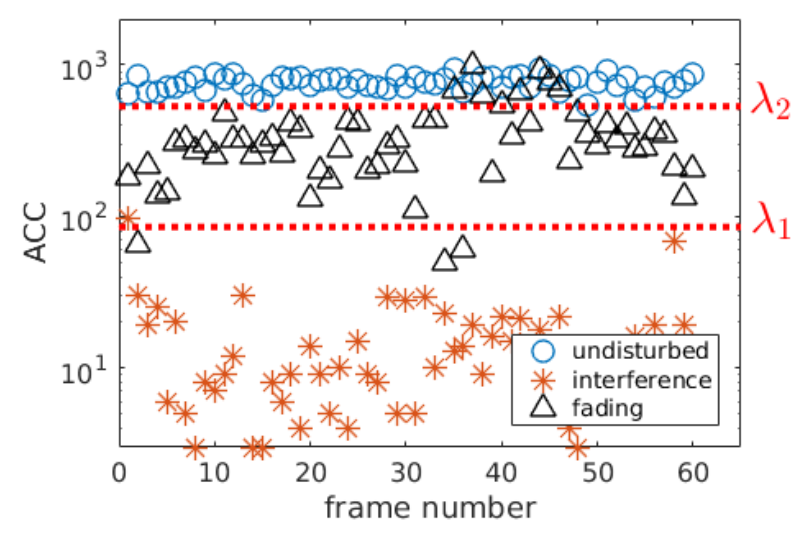

Fig. 8. Representation of the labelled input training set of ACC with the thresholds $\lambda_{1}$ and $\lambda_{2}$.

The authors also claim that multi-path fading and attenuation cannot be distinguished. Clearly, the results shown in Figure 8 show that, using our ACC as a channel state indicator, it is possible to distinguish these two states. To test the authors' first claim and compare the SED with our work, we reuse our acquisition dataset and compute a SED for each received frame. This is possible since the data contained in the acquired frames are encoded using the $1 / 2$ rate convolutional code defined in the LECIM FSK PHY.

First, we observe that the SED is zero when there are no errors in the frame, rendering it unusable in a medium SNR channel. This is a major limitation in a channel-aware LPWA system in which we specifically aim to have wireless links which have low to medium SNR. Since our dataset includes frames received at various SNR levels, we must therefore exclude all of these frames from the analysis. For the remaining frames, we observe that, whatever the channel class, all SED values tend to zero with increasing SNR, simply because errors become less common. Since a fixed threshold is used to distinguish between the two classes, distinguishing these two in the context of variable SNR experiments is thus impossible. SED will therefore be an adequate indicator only in very low SNR scenarios where the decoding algorithm corrects a sufficient number of errors. The channel state indicator proposed in this work does not suffer from this limitation. In conclusion, in an LPWA context, while our approach offers 3-state classification with $96 \%$ accuracy, the SED indicator offers only limited use and accuracy.

\section{CONCLUSION}

Accurate channel state identification is the cornerstone of lifetime enhancement of massive IoT wireless nodes based on LPWA communication schemes. This work proposes a low complexity channel state indicator computed directly within the DBB and which can easily be generalized to other LPWAN standards. This indicator is used to define a threestate classifier that achieves a correct decision rate of $96 \%$ when distinguishing 'undisturbed', 'fading' and 'interference' 
channels. The proposed indicator remains relevant at medium SNR, when the state-of-the-art SED indicator becomes unusable. Our work will aid in the development of cross-layer channel-aware adaptation strategies. Future work will include experimentation with non constant envelope interferers (e.g. Sigfox) and different propagation environments for the validation of the classifier performance in generalized scenarios.

\section{ACKNOWLEDGMENT}

The authors thank François Dehmas for fruitful discussions on DBB implementation as well as Olivier MICHEL and Pierre COMON for the provision of the RIESCO experimentation platform within GIPSA-LAB. This work was supported by the LabEx PERSYVAL-Lab (ANR-11LABX-0025-01).

\section{REFERENCES}

[1] N. Baccour, A. Kouba, L. Mottola, M. A. Ziga, H. Youssef, C. A. Boano, and M. Alves, "Radio link quality estimation in wireless sensor networks: A survey," ACM Transactions on Sensor Networks (TOSN), vol. 8, no. 4, p. 34, 2012.

[2] Kaishun Wu, Haoyu Tan, Hoi-Lun Ngan, Yunhuai Liu, and Lionel M. $\mathrm{Ni}$, "Chip Error Pattern Analysis in IEEE 802.15.4," IEEE Transactions on Mobile Computing, vol. 11, no. 4, pp. 543-552, Apr. 2012.

[3] C. Reis, R. Mahajan, M. Rodrig, D. Wetherall, and J. Zahorjan, "Measurement-based models of delivery and interference in static wireless networks," in ACM SIGCOMM Computer Communication Review, vol. 36. ACM, 2006, pp. 51-62.

[4] K. Jamieson and H. Balakrishnan, "Ppr: Partial packet recovery for wireless networks," SIGCOMM Comput. Commun. Rev., vol. 37, no. 4, pp. 409-420, Aug. 2007.

[5] Mythili Vutukuru, Hari Balakrishnan, and Kyle Jamieson, "Cross-layer wireless bit rate adaptation," ACM SIGCOMM Computer Communication Review, vol. 39, no. 4, pp. 3-14, 2009.

[6] Filip Barac, Stefano Caiola, Mikael Gidlund, Emiliano Sisinni, and Tingting Zhang, "Channel Diagnostics for Wireless Sensor Networks in Harsh Industrial Environments," IEEE Sensors Journal, vol. 14, no. 11, pp. 3983-3995, Nov. 2014.

[7] "IEEE Standard for Low Rate Wireless Networks-IEEE Std 802.15.42015."

[8] Ettus Research, "USRP Hardware Driver and USRP Manual-USRP2 and N2x0 Series." [Online]. Available: http://files.ettus.com/manual/ page_usrp2.html

[9] - "USRP Hardware Driver and USRP Manual-Daughterboards." [Online]. Available: http://files.ettus.com/manual/page_dboards.html\# dboards_wbx

[10] J. G. Proakis, Digital Communications, 4th ed. McGraw-Hill, 2000.

[11] V. Talla, M. Hessar, B. Kellogg, A. Najafi, J. R. Smith, and S. Gollakota, "LoRa Backscatter: Enabling The Vision of Ubiquitous Connectivity," Proceedings of the ACM on Interactive, Mobile, Wearable and Ubiquitous Technologies, vol. 1, no. 3, pp. 1-24, Sep. 2017. 